\title{
The Effect of Platelet-rich Plasma on Wounds of OLETF Rats Using Expression of Matrix Metallo- proteinase- 2 and -9 mRNA
}

\author{
Ho Seong Shin, Hwa Young Oh \\ Department of Plastic and Reconstructive Surgery, Soonchunhyang University Bucheon Hospital, Soonchunhyang University College of \\ Medicine, Bucheon, Korea
}

Background Complicated diabetic patients show impaired, delayed wound healing caused by multiple factors. A study on wound healing showed that platelet-rich plasma (PRP) was effective in normal tissue regeneration. Nonetheless, there is no evidence that when plateletrich plasma is applied to diabetic wounds, it normalizes the diabetic wound healing process. In this study, we have analyzed matrix metalloproteinase (MMP)-2, MMP-9 expression to investigate the effect of PRP on diabetic wounds.

Methods Twenty-four-week-old male Otsuka Long-Evans Tokushima Fatty rats were provided by the Tokushima Research Institute. At 50 weeks, wounds were arranged in two sites on the lateral paraspinal areas. Each wound was treated with PRP gel and physiologic saline gauze. To determine the expression of MMP-2, MMP-9, which was chosen as a marker of wound healing, reverse transcription polymerase chain reaction (RT-PCR) was performed and local distribution and expression of MMP-2, MMP-9 was also observed throughout the immunohistochemical staining.

Results RT-PCR and the immunohistochemical study showed that the levels of MMP-2, MMP-9 mRNA expression in PRP applied tissues were higher than MMP-2, MMP-9 mRNA expression in saline-applied tissues. MMP-9 mRNA expression in wounds of diabetic rats decreased after healing began to occur. But no statistical differences were detected on the basis of body weight or fasting blood glucose levels.

Conclusions This study could indicate the extracellular matrix-regulating effect observed with PRP. Our results of the acceleration of wound healing events by PRP under hyperglycemic conditions might be a useful clue for future clinical treatment for diabetic wounds.

Keywords Platelet-rich plasma / Rats, OLETF/ Matrix mtalloproteinase-2 / Matrix metalloproteinase-9

\author{
Correspondence: Ho Seong Shin \\ Department of Plastic and \\ Reconstructive Surgery, \\ Soonchunhyang University Bucheon \\ Hospital, Soonchunhyang University \\ College of Medicine, 170 Jomaru-ro, \\ Wonmi-gu, Bucheon 420-767, Korea \\ Tel: +82-32-621-5319 \\ Fax: +82-32-621-5662 \\ E-mail: shinerim@hanmail.net
}

This article has been adapted from Ho Seong Shin's dissertation submitted to Soonchunytang University Graduate School for the Ph.D. in Plastic Surgery.

This article was presented at the 68th Congress of The Korean Society of Plastic and Reconstructive Surgeons on November 4, 2010 in Seoul, Korea.

No potential conflict of interest relevant to this article was reported.

Received: 1 Oct 2011 • Revised: 1 Nov 2011 • Accepted: 2 Nov 2011

pISSN: 2234-6163 • elSSN: 2234-6171 • http://dx.doi.org/10.5999/aps.2012.39.2.106• Arch Plast Surg 2012;39:106-112

\section{INTRODUCTION}

The normal healing of a cutaneous wound is achieved via a complex biological and molecular process. Complicated diabetic patients show impaired, delayed wound healing caused by multiple factors including abnormalities of the biochemical environment and deficiency of growth factors [1]. Platelets are known to contain various factors involved in the repair of vasculature 
and tissues, and it is known that the specialized platelet secretary granules, alpha granules, contain platelet-derived growth factor, transforming growth factor-beta, epidermal growth factor, and others [2]. A study on wound healing also showed that plateletrich plasma (PRP) was effective in normal tissue regeneration [3]. Nonetheless, there have been no studies investigating whether platelet-rich plasma normalizes the wound healing process when applied to a diabetic wound. In this study, we have analyzed matrix metalloproteinase (MMP)-2, MMP-9 expression to investigate the effect of PRP on diabetic wounds using reverse transcription-polymerase chain reaction (RT-PCR) and immunofluorescent staining. MMP-9 is considered to play an important role in extracellular matrix remodeling. The wound healing process requires the deposition and accumulation of collagenous and noncollagenous extracellular matrix (ECM) by MMP [4]. We have correlated these data to the known effect of PRP on diabetic wound healing as well.

\section{METHODS}

\section{Animals and surgical procedures}

Otsuka Long-Evans Tokushima Fatty (OLETF) rats were provided by the Tokushima Research Institute (Otsuka Pharmaceutical, Tokushima, Japan). Twenty-four-weeks-old male OLETF rats were housed singly with food and water, were given free access to rat chow, and were kept in a controlled temperature $\left(23 \pm 2^{\circ} \mathrm{C}\right)$ and humidity $(55 \pm 5 \%)$ under an artificially controlled light cycle [5]. At 50 weeks, the mean body weight and fasting blood glucose were $763.40 \pm 56.05 \mathrm{~g}$ and $409.70 \pm 42.02$ $\mathrm{mL} / \mathrm{dL}$. At 50 weeks, the dorsal hair was removed with hair clippers and the skin cleaned with chlorhexidine. A template was used to define the wound sites, which were organized into two sites on the lateral paraspinal areas. Before wounding, each wound site was demarcated with a sterile ink and template and a No. 15 blade was used to excise a $1.75-\mathrm{cm}^{2}$ wound, taking care not to injure the underlying subcutaneous layers, and covered with moist gauze to avoid desiccation. The wounds had an average depth of approximately $3.4 \pm 1 \mathrm{~mm}$. Each rat had two wounds created on the left and right sides. The each wound of the left side was treated with PRP gel, and the wound of right side was treated with physiologic saline gauze. Fig. 1 shows the wound placement and wounding patterns used. The dorsal wounds were then covered with adherent film dressing (Tegaderm). All animals received cephalosporin $(50 \mathrm{mg} / \mathrm{kg})$ at 30 minutes before wounding. The wounds were examined on postwounding day $1,2,3,5$, and 7 ( 4 animals/day). A total 40 tissue samples were obtained from 20 OLETF rats who had undergone wounding.
Fig. 1. Wound placement and wounding patterns used

Full thickness wounds were located on paraspinal skin regions of both sides of the OLETF rat. Wound size was $1.5 \times 1.5 \mathrm{~cm}$. Distance of between $A$ and $B$ wounds were $2 \mathrm{~cm}$. The average depth of each wound was $3.4 \pm 0.4 \mathrm{~mm}$. Platelet-rich plasma applied site $A$, saline applied site $B$.

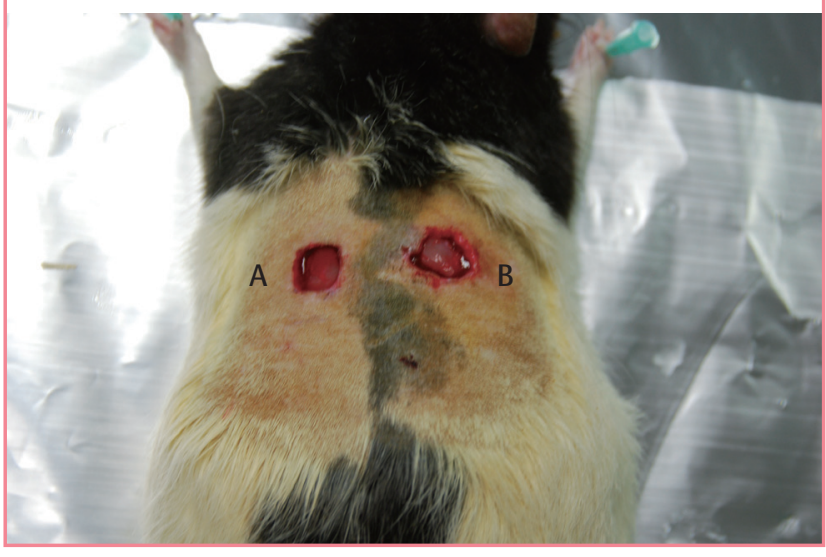

\section{Preparation of platelet-rich plasma}

PRP was prepared from the blood of 6 rats. The donor rats were anesthetized, and their blood was collected by cardiac puncture under anesthesia. The animals were killed by an intracardiac injection of an overdose of pentobarbital sodium. The whole 120$\mathrm{mL}$ of blood was added immediately to an anticoagulant. Citrate phosphonate dextrose (CPD) buffer $(0.15 \mathrm{mg}, \mathrm{CPD} \mathrm{mL})$ was added in a ratio of $1 \mathrm{~mL}$ of CPD buffer to $5 \mathrm{~mL}$ of blood. The blood was then centrifuged at $220 \mathrm{G}$ for 20 minutes. The supernatant was saved to produce fibrin glue. The precipitate containing PRP was used for a second centrifugation at $480 \mathrm{G}$ for 20 minutes. The pellet from the second centrifugation was saved and diluted with supernatant until the platelet concentration became $900,000 / \mu \mathrm{L}$. This platelet concentration was 3.9 times more than mean platelet count of the donor rats. To reduce the risk of a graft-versus-host reaction, the platelet concentrate and supernatant were irradiated at $25 \mathrm{~Gy}$ according to international blood banking standards to inactivate the white blood cells. Purified bovine thrombin (thrombin, Sigma-Aldrich, Munich, Germany) was diluted and buffered in a solution of $10 \mathrm{~mL} 10 \%$ calcium chloride to obtain a concentration of $100 \mathrm{U} / \mathrm{mL}$.

\section{RT-PCR of MMP-2, MMP-9 mRNA}

Total RNA was isolated from the tissues of wounds using Trizol reagent (Invitrogen, Carlsbad, CA, USA) according to the manufacturer's instructions. To eliminate genomic DNA from the samples, DNase I treatment (Qiagen, Hilden, NorthRhine-Westphalia, Gemany) was included in the RNA isolation procedure. The cDNA was prepared using a Superscript II kit (Invitrogen) and used as a template for analysis of gene expression by PCR. The 
Table 1. The primer sequences of matrix metalloproteinase (MMP)-2, MMP-9 mRNA

\begin{tabular}{|c|c|c|}
\hline Name & Sequence & $\begin{array}{l}\text { Product size } \\
\text { (bp) }\end{array}$ \\
\hline \multicolumn{3}{|l|}{$\beta$-actin } \\
\hline Forward & 5'-GGACTTCGAGCAAGAGATGG-3' & 233 \\
\hline Reverse & 5'-AGCACTGTGTTGGCGTACAG-3' & \\
\hline \multicolumn{3}{|l|}{ MMP-2 } \\
\hline Forward & 5'-AGACCGCCATGTCCACTGTT-3' & 131 \\
\hline Reverse & 5'-TGGTCGCACACCACATCTTT-3' & \\
\hline \multicolumn{3}{|l|}{ MMP-9 } \\
\hline Forward & 5'-AAGATGCTGCTGTTCAGCGGG-3' & 254 \\
\hline Reverse & 5'-GTCCTCAGGGCACTGCAGGAT-3' & \\
\hline
\end{tabular}

RNA was reverse transcribed by incubation with $10 \mathrm{mMd}$ NTP, $1 \mu \mathrm{L}$ oligo and $1 \mu \mathrm{L}$ SuperScript II $(200 \mathrm{U} / \mu \mathrm{L}$, Life Technologies, Grand Island, NY, USA) at $42^{\circ} \mathrm{C}$ for 50 minutes, and then heatinactivated at $70^{\circ} \mathrm{C}$ for 15 minutes. After reverse transcription, the cDNA was divided into aliquots in tubes that contained specific primer pairs for the mouse MMP-2, MMP-9 genes. Primers and probes were designed for the selected genes by GeneFisher software (http://bibiserv.techfak.uni-bielefeld.de/genefisher2) (Table 1). The following primer sequences (shown $5^{\prime} \rightarrow 3^{\prime}$ ) were used MMP-2: AGA CCG CCA TGT CCA CTG (forward), TGG TCG CAC ACC ACA TCT (reverse) and MMP-9: AAG ATG CTG CTG TTC AGC GGG (forward), GTC CTC AGG GCA CTG CAG GAT (reverse). RT-PCR was performed in a GeneAmp PCR system 9600 (Perkin-Elmer Applied Biosystem, Applied Biosystems, Foster City, CA, USA) with an initial denaturation step of $94^{\circ} \mathrm{C}$ for 5 minutes, followed by 35 cycles of 1 minute at $62^{\circ} \mathrm{C}$ (MMP-9), 1 minute at $72^{\circ} \mathrm{C}$, and a final extension at $72^{\circ} \mathrm{C}$ for 7 minutes gene expression was measured in 40 independent skin tissues of OLETF rats. The amplified PCR products were electrophoresed on $1 \%$ agarose gel and visualized by ethidium bromide staining, together with the 100-bp DNA ladder marker (Bioneer Co., Daejeon, Korea). Sequencing of PCR products was performed using PCR amplicon Sequencing. The PCR products of the MMP-2, MMP-9 genes were mixed with PCR primer and sequenced using the ABI BigDye Terminator Cycle Sequencing Kit (Applied Biosystems) and an ABI PRISM 3700 DNA analyzer (Applied Biosystems). The derived sequences were compared with those of other genes using the Basic Local Alignment Search Tool program of the National Institutes of Health.

\section{Immunohistochemical staining}

The paraffin sections $(4-\mu \mathrm{m})$ were deparaffinized in xylene and rehydrated. First, each slide prepared with 4- $\mu \mathrm{m}$ thick sections of embedded tissue was deparaffinized. The tissue was subjected to hematoxylin and eosin ( $\mathrm{H} \& \mathrm{E})$ staining to permit morpho- metric analysis. For immunohistochemistry of MMP-2, MMP9 , the tissue on slides was treated with $0.3 \% \mathrm{H}_{2} \mathrm{O}_{2}$ for 20 minutes to block endogenous peroxidase, and then incubated at $4^{\circ} \mathrm{C}$ overnight with anti-MMP-2 mouse antibody (SC-13595, 1:50 dilution, Santa Cruz Biotechnology Inc., Santa Cruz, CA, USA) and anti-MMP-9 antibody (SC-6840, 1:50 dilution, Santa Cruz Biotechnology Inc.). After that, the slides were incubated with an ABC kit (Vector Laboratories, Burlingame, CA, USA). The color was developed with 3.3' -disminobenzidine tetrachloride (Zymed Laboratory Inc., San Francisco, CA, USA). The slides were observed using a light-microscope adhered to a chargedcoupled camera device (Pulnix, Andover, MA, USA).

\section{Assessment of immunohistochemical staining}

In immunohistochemical evaluation, the histochemical score (HSCORE) was used for comparison and standardization. The HSCORE was calculated using the following equation: HSCORE $=\Sigma \mathrm{Pi}(\mathrm{i}+1)$, where $\mathrm{i}$ is the intensity of staining with a value of 0,1 or 2 ( 0 , weak staining; 1 , moderate staining; or 2 , strong staining) and $\mathrm{Pi}$ is the percentage of stained cells varying from $0 \%$ to $100 \%(0.0-1.00)$. The HSCOREs ranged from a minimum of zero in cases with no staining to a maximum of 3.0. The HSCORE of MMP-2, MMP-9 were determined by two sets of independent investigators.

\section{Statistical analysis}

Statical analysis was performed with SPSS ver. 14.0 (SPSS Inc., Chicago, IL, USA). The immunochemical studies were analyzed using the nonparametric Mann-Whitney test, All data are expressed as median values and $\mathrm{P}<0.05$ was considered to be statistically significant.

\section{RESULTS}

\section{RT-PCR analysis}

Signals for MMP-2 mRNA were seen to be expressed in PRP applied tissues at 1, 3 days after treatment with maximal expression being seen at first day corresponding physiologic saline applied tissues and the intensity of optical density (IOD) ranged from 20,105 to 5,822. Respectively, MMP-2 mRNAs showed a decrease following day 1 to 7. Signal for MMP-2 mRNA were detected in physiologic saline-applied tissues on 3, 5 day after treatment. The levels of MMP-2 mRNA in PRP applied tissues were positively related to postwounding days, whereas MMP2 mRNA expression in saline-applied tissues remained on 5 day after treatment (Fig. 2). No statistical difference was detected on the basis of body weight, fasting blood glucose levels.

MMP-9 mRNA was undetectable in saline-applied tissues, 
Fig. 2. Matrix metalloproteinase-2 mRNA expression profile during acute healing period

PRP, platelet-rich plasma.

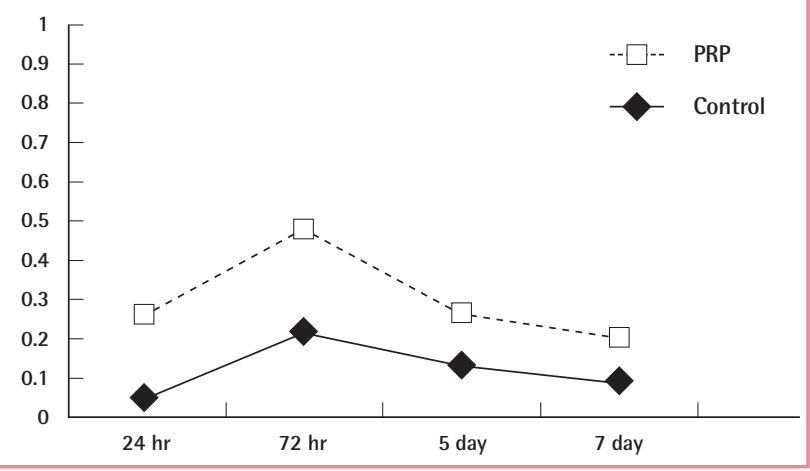

Fig. 3. PRP on mRNA expression of MMP-9 in RT-PCR

PRP, platelet-rich plasma; MMP, matrix metalloproteinase; RT-PCR, reverse transcription polymerase chain reaction.

$254 \mathrm{bp}$

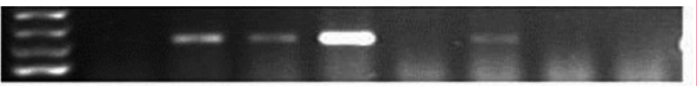

$\beta$-actin

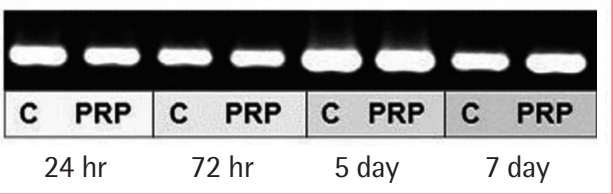

except at 3 days after treatment. Following PRP-application MMP-9 mRNA expression was detected, with maximal expression being seen on the third day (Fig. 3). The levels of MMP-9 mRNA in PRP applied tissues were reported high intensity of optical density related to saline applied tissues. The IOD ranged from 33,698 to 955. MMP-9 was especially up-regulated where it was expressed in PRP applied wounds with mild positivity on the first day. The saline applied tissues had returned to no expression on day 7, but the PRP-applied tissues were weakly positive expression on day 7 (Fig. 4). No statistical difference was detected on the basis of body weight or fasting blood glucose levels.

\section{H\&E staining examination}

Morphological analysis by $H \& E$ staining of tissues was performed on all the tissues (Fig. 5). Tissue sections for each time point were also analyzed to determine the epithelial tongue area and granulation tissue area. One day after injury, red blood cells and dilatation of blood vessels were observed. Acute inflammatory cells, such as neutrophils, infiltrated the wound edge area. Three days after injury, the inflammatory cells decreased, and a large number of fibroblast-like cells, polygonal-shaped cells, and new blood vessels were found in the upper dermal area. Seven days after injury, one to two layers of well-arranged keratinocyte-
Fig. 4. Matrix metalloproteinase-9 mRNA expression profile during acute healing period

PRP, platelet-rich plasma.

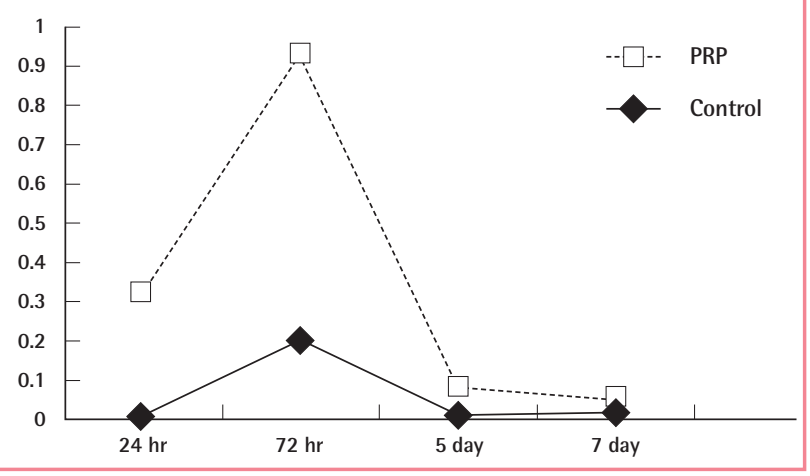

Fig. 5. Postwounding tissues following 3 days after treatment

(A) Red blood cells and dilatation of blood vessels were observed. Acute inflammatory cells, such as neutrophils, infiltrated in wound edge area in platelet-rich plasma-applied group. (B) Inflammatory cell decreased in the upper dermal area in saline-applied group $\left(H \& E_{1}\right.$ $\times 100$ ).
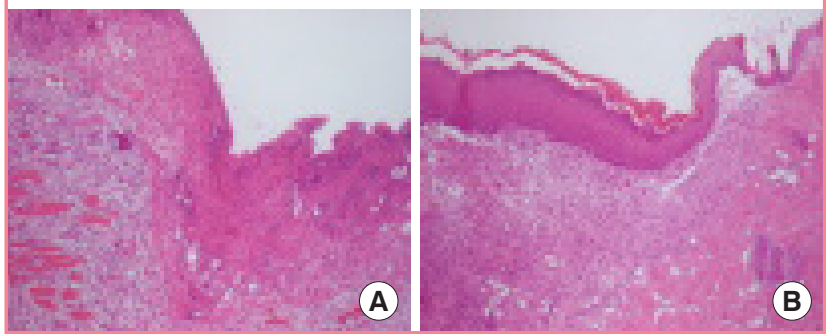

like cells were surrounded by immature collagen matrix at the edge of the tissues.

\section{Immunohistochemical examination}

The immunohistochemical stain for MMP-2 protein in tissue samples of PRP-appled wounds showed dark brown pigmentation on the first day. MMP-2 expression in the two groups of tissue was significantly decreased following wounding. MMP-2 localization was identical on days 1, 3, and 5 in PRP-applied tissues and identical on days 1,3 , and 5 in saline-applied tissues.

MMP-9 staining is shown in Fig. 6. Indirect immunofluorescence was used to localize MMP-9 protein within tissues. At day 1, MMP-9 staining was weak in all tissues. At day 3, MMP9 staining was observed to be diffuse positive and decreasing in intensity further away from the wound, whereas at day 7 the staining was more concentrated along the wound edge, with only minor staining being seen away from the wound in PRPapplied tissues. It was found that in PRP-applied tissues, the intensity of MMP-9 was significantly up-regulated at day 1 and 3 rather than in the saline-applied tissues. In addition, the inten- 


\section{Fig. 6. MMP-9 protein following 3 days after treatment}

Finding of platelet-rich plasma-applied wounds showed more darker brown pigmentation than control in matrix metalloproteinase (MMP)-9 protein expression. (A) MMP-9. (B) Control (Immunochemical stain, $\times 100$ ).
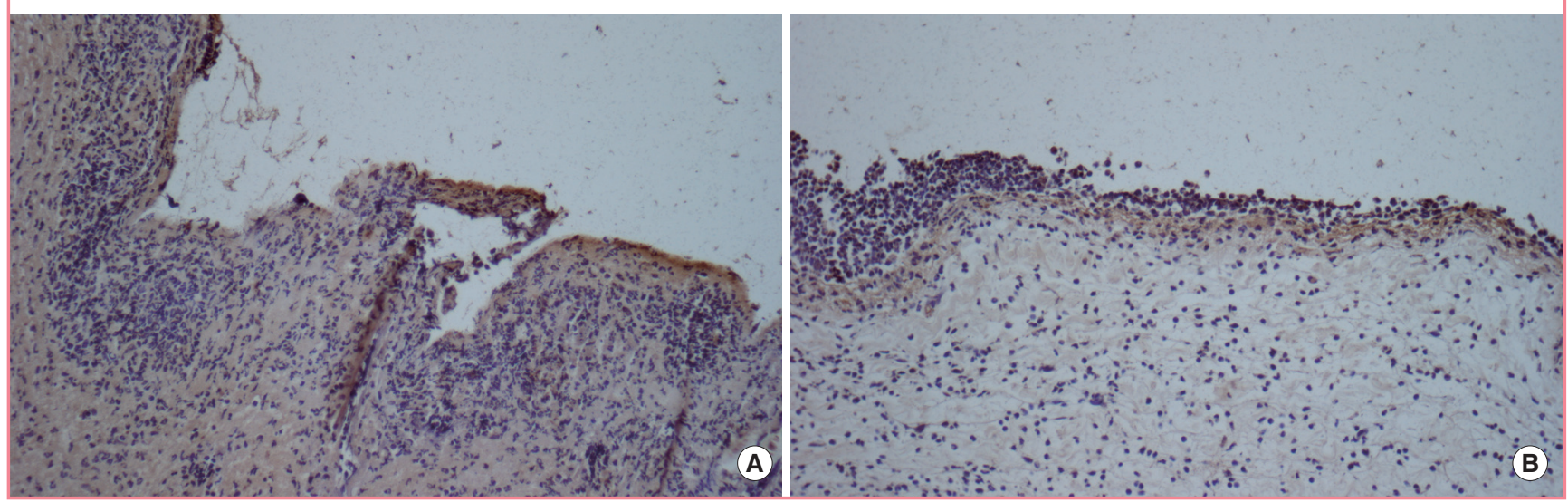

Table 2. Statistical analysis of MMP-9 protein immunohistochemical staining

\begin{tabular}{|lcc|}
\hline & Mean \pm SD & P-value \\
\hline 24 hr & $1.425 \pm 0.3304$ & 0.029 \\
PRP & $0.850 \pm 0.1000$ & \\
Control & $1.600 \pm 0.1633$ & 0.014 \\
72 hr & $0.750 \pm 0.1915$ & \\
PRP & \\
Control & $0.600 \pm 0.1414$ & 0.886 \\
5 day & $0.575 \pm 0.1708$ & \\
PRP & & \\
Control & $0.425 \pm 0.1708$ & \\
7 day & $0.475 \pm 0.0957$ & \\
PRP & \\
Control & & \\
\hline MMP, matrix metalloproteinase; SD, standard deviation; PRP, platelet-rich plasma. \\
\hline
\end{tabular}

sity of MMP-9 protein was no different in the two groups of tissues after 7 day. The HSCORE of MMP-9 for the control group ranged from 0.20 to 1.20 with the average at 0.65 (standard deviation $=1.359$ ). Statisitical differences in the MMP-9 protein between PRP group and control group were found on the first and third day after treatment (Table 2). The immunohistochemical study showed results similar to that of the RT-PCR analysis for MMP-9. However, there were no significant differences in the HSCORE between the two groups at 5 and 7 days after treatment $(\mathrm{P}>0.05)$.

\section{DISCUSSION}

This study demonstrates the potency of PRP in promoting hyperglycemic conditioned wound healing. PRP is defined as a portion of the plasma fraction of autologous blood having a platelet concentration above baseline [6]. As such, platelet-rich plasma contains not only a high level of platelets but also the full complement of clotting factors; the regenerative potential of PRP depends, to a large extent, on the levels of secretary proteins that are released on platelet activation. These protein levels will depend on several factors, including 1) the concentrations of these proteins contained in the platelets and 2) the processing technique, which will influence platelet concentration and whether platelets are activated or fragmented during preparation [7]. Proponents of plateletrich plasma technology suggest that benefits of the completeness of platelet activation before measurement include an increase in hard and soft tissue wound healing and decrease in postoperative infection, pain, and blood loss [8].

There have been numerous publications on the use of PRP for several clinical applications. Although the vast majority of these studies have yielded excellent outcomes, most of the case studies were limited to nondiabetics. Mathew et al. found adipose stem cells combined with PRP contributed to higher quantities of arteioles formed in healed wounds treated with these agents [9]. Clinically, it is well known that wounds with poor vascularity heal poorly. Marx et al. [10] reported enhanced bone formation in mandibular bone grafts treated with PRP, an effect lasting for 6 months postoperatively that was documented by biopsy enhanced bone deposition among PRP-treated grafts. Much evidence has indicated that high blood glucose could directly contribute to poor wound healing and ulceration formation in diabetes mellitus. However, the first independent assessment of the efficacy of PRP on diabetic wounds was reported recently by Margolis et al. [11]. They found that when compared with standard care alone, using PRP improves the environment of diabetic foot ulcers so that they heal after 32 weeks of care. They reported that PRP also appears to be effective in healing severe diabetic wounds and may, therefore, play an important role in preventing amputation. However, there is currently no final con- 
sensus on the effect of PRP on diabetic wounds. The goal of our study was to estimate the effectiveness of PRP on hyperglycemic wounds using expression of MMP-2, MMP-9 mRNA.

The interactions of cells with ECM are critical for the normal wound healing process [12]. The turnover and remodeling of ECM must be highly regulated since undercontrolled proteolysis contributes to an abnormal wound healing process and to the generation of many pathological conditions characterized by either abnormal degradation ordeposition of ECM components. MMPs are a major group of enzymes that regulate cell-matrix composition. The MMPs are zinc-dependent endopeptidases known for their ability to cleave one or several ECM constituents, as well as nonmatrix proteins. The reason for this histopathologically dependent expression profile of the gene may be put in relation to the major biological predictor of hyperglycemic conditioned wounds after PRP treatment. MMP-9 is the finest marker of hyperglycemic wound healing. Thus we chose to use local expression of MMP in the wound edges.

Some studies have reported that diabetic ulcers are characterized by increased MMP [13]. These studies have found that protease levels in chronic wound fluid from diabetic patients are elevated compared with healing wound fluid and that protease levels of wounds decrease after healing begins to occur. Most of these studies have examined protease levels in wound fluid as an indicator of tissue protease levels. However, we have observed that local expression of MMP-2, MMP-9 wasless detectable in diabetic rats than nondiabetic rats in an experiment. The rat model did not show similar alterations in MMP expression as did the human model. Furthermore, in vitro studies (human fibroblast and human keratinocyte studies) also did not show similar alterations in MMP expression in human models.

Our study showed relatively low expression of MMP-2 mRNA than MMP-9 in early healing periods, these results suggested that MMP-2 contribute to healing of wound more later than MMP-9. Differencies in MMP-2 expression were not significantly observed between two groups, the cause of these results was that short period OLETF animal model could not illustrate delayed MMP-2 activation. The monocyte and macrophage is the main MMP-9 expressing cell within the wound. The neutrophils are also known to store but not synthesize it. Previous studies have localized MMP-9 mRNA to basal keratinocytes near the epidermal migrating front and deep within the granulation tissue [14]. In this study, MMP-9 protein showed greater dermal staining than in previously published work. MMP-9 may be involved in kerationocyte detachment from the basement membrane before migration and aid matrix digestion by neutrophils and macrophages during removal of necrotic tissue from the wound. The reduced levels of MMP-9 in diabetic rats are probably a reflec- tion of this delayed recruitment. Significant differences in MMP9 expression were observed between wounds applied with PRP and those applied with physiological saline. Treatment with PRP results in a significant increase in MMP-9 when looking at early wounds at 1 and 3 days after treatment. The density of MMP-9 mRNA expression and immunohistochemical findings of MMP-9 were similar.

This information suggests that PRP is a reliable initiator of healing. These data, obtained for the first time in RT-PCR approaches, are in agreement with the recent data obtained with immunohistochemical staining for MMP-9, showing an association between its expression and diabetic rat wound healing rates [15]. In addition, we also observed that MMP-9 expression represents an independent recruitment marker, as recently stated. However, we found a good correlation with some of the most common clinical features in the wounds of diabetic rats. MMP9 expression was significantly related to wound status [16]. Our data seem to demonstrate a simultaneous and coordinated upregulation of MMP-9 expression at the mRNA level, in hyperglycemic conditioned wounds even if this phenomenon seems variable according to the blood glucose level.

The general limitationw of our investigation are the following: 1) the sample sizewas inadequate; otherwise multivariate analysis would have determined which protease levels are independently associated with diabetic wound environments, 2) spontaneously diabetic rodent models such as OLETF rats cannot stimulate the metabolic abnormalities of the human diabetic wounds correctly. The characteristics of OLETF rats include: 1) late onset of hyperglycemia after 18 weeks of age, 2) mild obesity, 3) multiple recessive genes that are involved in the induction of diabetes. Hyperglycemia is necessary to stimulate diabetic wounds, but not sufficient alone for the development of multifactorial diabetic chronic wounds. Nevertheless, there have been no reports on the direct topical application of PRP on a hyperglycemic conditioned experimental wound model thus far.

In summary, our study suggests that platelet-rich plasma not only contributes to delivery growth factors but also recruits inflammatory cells that exceed those infiltrated by the body, thereby accelerating the healing process. Complete understanding of these effects and validation of this new treatment modality will be possible through our current studies of these treatment combinations in diabetic animal models.

\section{REFERENCES}

1. Falanga V. Wound healing and its impairment in the diabetic foot. Lancet 2005;366:1736-43.

2. Anitua E. Plasma rich in growth factors: preliminary results 
of use in the preparation of future sites for implants. Int J Oral Maxillofac Implants 1999; 14:529-35.

3. Marx RE. Platelet-rich plasma: evidence to support its use. J Oral Maxillofac Surg 2004;62:489-96.

4. Madlener M, Parks WC, Werner S. Matrix metalloproteinases (MMPs) and their physiological inhibitors (TIMPs) are differentially expressed during excisional skin wound repair. Exp Cell Res 1998;242:201-10.

5. Kawano K, Hirashima T, Mori S, et al. Spontaneous longterm hyperglycemic rat with diabetic complications. Otsuka Long-Evans Tokushima Fatty (OLETF) strain. Diabetes 1992;41:1422-8.

6. Simman R, Hoffmann A, Bohinc RJ, et al. Role of plateletrich plasma in acceleration of bone fracture healing. Ann Plast Surg 2008;61:337-44.

7. Man D, Plosker H, Winland-Brown JE. The use of autologous platelet-rich plasma (platelet gel) and autologous platelet-poor plasma (fibrin glue) in cosmetic surgery. Plast Reconstr Surg 2001; 107:229-37.

8. Della Valle A, Sammartino G, Marenzi G, et al. Prevention of postoperative bleeding in anticoagulated patients undergoing oral surgery: use of platelet-rich plasma gel. J Oral Maxillofac Surg 2003;61:1275-8.

9. Blanton MW, Hadad I, Johnstone BH, et al. Adipose stromal cells and platelet-rich plasma therapies synergistically increase revascularization during wound healing. Plast Reconstr Surg 2009; 123:56S-64S.

10. Marx RE, Carlson ER, Eichstaedt RM, et al. Platelet-rich plasma: Growth factor enhancement for bone grafts. Oral Surg Oral Med Oral Pathol Oral Radiol Endod 1998;85:638-46.

11. Margolis DJ, Kantor J, Santanna J, et al. Effectiveness of platelet releasate for the treatment of diabetic neuropathic foot ulcers. Diabetes Care 2001;24:483-8.

12. Medina A, Scott PG, Ghahary A, et al. Pathophysiology of chronic nonhealing wounds. J Burn Care Rehabil 2005;26: 306-19.

13. Liu Y, Min D, Bolton T, et al. Increased matrix metalloproteinase-9 predicts poor wound healing in diabetic foot ulcers. Diabetes Care 2009;32:117-9.

14. Lan CC, Liu IH, Fang AH, et al. Hyperglycaemic conditions decrease cultured keratinocyte mobility: implications for impaired wound healing in patients with diabetes. Br J Dermatol 2008;159:1103-15.

15. Lund LR, Romer J, Bugge TH, et al. Functional overlap between two classes of matrix-degrading proteases in wound healing. EMBO J 1999;18:4645-56.

16. Wall SJ, Bevan D, Thomas DW, et al. Differential expression of matrix metalloproteinases during impaired wound healing of the diabetes mouse. J Invest Dermatol 2002;119:91-8. 